\title{
Über Paare von Limitierungsverfahren
}

\author{
KONRAD KNOPP zum Gedächtnis \\ Von
}

\section{G. G. LORENTZ und K. ZELLER}

\section{Einleitung}

Eine Folge $x=\left\{x_{k}\right\}$ heißt limitierbar durch das Matrixverfahren (oder kürzer: die Matrix, das Verfahren) $A$ zum Werte $a=A-\lim x$, wenn alle Zahlen

$$
y_{l}=x_{l}^{A}=x_{l}(A)=\sum_{k=0}^{\infty} a_{l k} x_{k} \quad(l=0,1, \ldots)
$$

im gewöhnlichen Sinne existieren und eine gegen $a$ konvergente Folge bilden.

Das Wirkfeld $\mathfrak{A}$ einer Matrix $A$ besteht aus allen $A$-limitierbaren Folgen. Ein Verfahren $A$ ist permanent- (oder regulär), wenn es jede konvergente Folge zu ihrem gewöhnlichen Grenzwert limitiert. Zwei Verfahren $A, B$ heißen verträglich, wenn sie niemals ein und derselben Folge verschiedene Grenzwerte zuordnen. Das Verfahren $A$ heiBt stärker als $B$ (in Zeichen $A>B$ ), wenn es alle $B$-limitierbaren Folgen limitiert. Man beachte, da $B$ hier Verträglichkeit nicht verlangt und die Möglichkeit $\mathfrak{U}=\mathfrak{B}$ zugelassen wird.

Manchmal betrachten wir nur beschränkte Folgen $x=\left\{x_{k}\right\}$. Diese bilden einen Banach-Raum $(m)$ mit der Norm $\|x\|=\sup _{k}\left|x_{k}\right|$ (außer in Abschnitt 8 wird $\|x\|$ immer diese Norm bedeuten). Mit $\mathfrak{A}_{B}$ bezeichnen wir das Wirkfeld von $A$ in $(m)$, d.h. den Durchschnitt $\mathfrak{A} \cap(m)$. Es ist klar, was z. B. die Feststellung bedeutet: „A und $B$ sind verträglich für beschränkte Folgen."

Wir benützen auch einige_Begriffe betreffend starke Limitierbarkeit und Kerne von Folgen. Eine Folge $x$ heißt stark limitierbar durch das Verfahren $A$ zum Werte $a$, wenn die in (2) auftretenden Zahlen für $l=0,1, \ldots$ existieren und

$$
\sum_{k=0}^{\infty} a_{l k}\left|x_{k}-a\right| \rightarrow 0 \quad(l \rightarrow \infty)
$$

gilt. Bei permanentem, positivem $A$ ist der starke $A$-Grenzwert von $x$ eindeutig bestimmt (falls er existiert). Zwei Matrizen $A, B$ sind stark verträglich, wenn aus starker $A$-Limitierbarkeit der Folge $x$ zum Werte $a$ und.starker $B$-Limitierbarkeit von $x$ zu $b$ die Gleichheit $a=b$ folgt.

Der Kern einer Folge $x=\left\{x_{k}\right\}$ ist der Durchschnitt aller abgeschlossenen konvexen Mengen in der komplexen Ebene, die höchstens endlich viele der $x_{k}$ nicht enthalten. Existieren in (1) alle $y_{l}$, so wird der Kern von $y$ auch der $A$-Kern von $x$ genannt. Zwei (permanente) Verfahren $A$ und $B$ heißen kernverträglich für beschränkte Folgen, wenn für jedes beschränkte $x \operatorname{der} A$-Kern 
und der $B$-Kern einen nichtleeren Durchschnitt besitzen (LoRENTZ-RoBiNSON [5] benützen eine andere Terminologie). Von zwei (permanenten) Matrizen $A$ und $B$ sagen wir, daß $B$ für beschränkte Folgen kern-stärker als $A$ ist, wenn der $B$-Kern jeder beschränkten Folge $x$ in deren $A$-Kern enthalten ist.

Eine permanente Matrix kann nicht zu stark sein, z.B. kann sie nicht alle beschränkten Folgen limitieren. Daher benützt man häufig mehrere Limitierungsverfahren $A, B, \ldots$ gleichzeitig (gewöhnlich wird man die Verträglichkeit dieser Verfahren annehmen). Das führt zu den folgenden Fragen. Sei $\mathfrak{A}+\mathfrak{B}+\cdots$ der von endlich vielen Wirkfeldern $\mathfrak{A}, \mathfrak{B}, \ldots$ aufgespannte lineare Raum, d.h. die Menge aller Folgen $z=x+y+\cdots$ mit $x \in \mathfrak{A}, y \in \mathfrak{B}, \ldots$. Wie groß kann die Menge $\mathfrak{Q}+\mathfrak{B}+\cdots$ sein ? Kann sie z. B. alle beschränkten Folgen umfassen?

Fragen dieser Art wurden aufgeworfen und teilweise beantwortet von Petersen (und Goffman) [7]. Unter anderem konstruiert Petersen zwei für beschränkte Folgen verträgliche, positive, permanente Matrizen $A$ und $B$, für die es kein positives permanentes $C$ gibt, das für beschränkte Folgen stärker als $A$ und $B$ ist. In dieser Arbeit geben wir allgemeinere Resultate in dieser Richtung (Aussagen über Existenz oder Nichtexistenz eines stärkeren $C$ ).

In Satz 1 weisen wir zwei permanente verträgliche Matrizen $A$ und $B$ auf, zu denen es kein permanentes $C$ mit $C>A, C>B$ gibt; was darauf beruht, da $B A$ und $B$ sehr schnell wachsende Folgen limitieren. Der Beweis benützt Ergebnisse von Erdös-PirÁnIan [4] über das verschiedene Verhalten von zeilenfiniten und nichtzeilenfiniten Matrizen. Das Beispiel zeigt, da $B$ gewisse Einschränkungen notwendig sind, um positive Resultate zu erhalten. Viele Möglichkeiten stehen hier offen (Betrachtung von zeilenfiniten Matrizen, von Matrizen mit bestimmten Perfektheitseigenschaften, von langsam wachsenden Folgen). In der vorliegenden Arbeit geben wir uns mit der Behandlung beschränkter Folgen zufrieden. Nur Abschnitt 8 (starke Limitierbarkeit) ist ein erster Schritt zur Untersuchung auch unbeschränkter Folgen.

Eigentümlicherweise benötigen wir jedoch für ein positives Ergebnis über Folgen in (m) Zusatzbedingungen, die unbeschränkte Folgen betreffen. Daß solche Bedingungen wesentlich sind, zeigt Satz 2, der ein Beispiel zweier permanenter Matrizen $A$ und $B$ gibt, die für beschränkte Folgen verträglich sind, bei denen es jedoch keine stetige Linearform auf $(m)$ gibt, die sowohl mit $A-\lim x$ als auch mit $B-\lim x$ übereinstimmt (in $\mathfrak{H}_{B}$ bzw. $\mathfrak{B}_{B}$ ). Das beruht auf dem Umstand, da $\beta A$ und $B$,fast unverträglich" sind, d.h. gewisse im Sinne der Norm von $(m)$ benachbarte Folgen zu ganz verschiedenen Werten limitieren [Singularität $\left(S_{1}\right)$ aus Abschnitt 3]. Bei der Konstruktion von $A$ und $B$ benützen wir einen Gedanken von PeTERSEN [ 7$]$, um verträgliche Paare von Matrizen 'mit „embryonaler Fast-Unverträglichkeit" zu finden, und kondensieren dann diese Eigenschaft zu "Fast-Unverträglichkeit".

Die beiden Matrizen in Satz 2 müssen jedoch für gewisse unbeschränkte Folgen unverträglich sein (Satz 3). Das trifft sogar zu, wenn die Singularität $\left(S_{1}\right)$ durch eine schwächere Singularität $\left(S_{2}\right)$ ersetzt wird (s. Abschnitt 5 , 
Lemma 2). Beim Beweis wird eine Folge $x$, bei der die Unverträglichkeit auftritt, mittels Kondensation von Singularitäten konstruiert, wobei noch ein Glättungsprözeß nötig ist.

Ein positives Ergebnis erhalten wir schließlich in Satz 4. Wenn zwei permanente Matrizen $A, B$ für alle Folgen verträglich sind, dann gibt es ein permanentes. $C$, das für alle beschränkten Folgen stärker als (und verträglich mit) $A$ und $B$ ist. Beim Beweis benötigen wir Lemma 2, das auf gewisse konvexe Mengen führt; Trennungssätze für konvexe Mengen erlauben die Konstruktion von $C$. Nachträglich bemerkten wir, daß eine ähnliche Methode von BRUDNo [3] benützt wurde, um die von ihm in [2], [3] eingeführten Normen von Wirkfeldern zu charakterisieren.

Die Lage ist etwas einfacher bei starker Summierbarkeit (wo wir auch unbeschränkte Folgen zulassen) und Kernvergleich (bezüglich beschränkter Folgen). Hier sind Verträglichkeitseigenschaften yon $A$ und $B$ notwendig und hinreichend für die Existenz eines stärkeren Verfahrens $C$. Der Beweis unseres Ergebnisses über Kerne (Satz 6) beruht auf der Trennung konvexer Mengen im Raume ( $\left.l^{1}\right)$, zu dem die Zeilen permanenter Matrizen gehören. Diesen Kunstgriff findet man schon bei LORENTZ und RoBINson [5] $\left.{ }^{1}\right)$.

\section{Nichtexistenz eines stärkeren Verfahrens}

Wir geben ein Beispiel, das teilweise die bei den späteren positiven Ergebnissen vorgenommene Einschränkung auf beschränkte Folgen oder starke Limitierbarkeit rechtfertigt.

Satz 1. Es gibt zwei permanente verträgliche Matrizen $A$ und $B$, so daß keine permanente Matrix $C$ gleichzeitig $C>A$ und $C>B$ erfüllt.

Beweis. Wir setzen

$$
A=\left(\begin{array}{ccccccc}
\frac{1}{2} & \frac{1}{2} & 0 & 0 & 0 & 0 & \ldots \\
0 & 0 & \frac{1}{2} & \frac{1}{2} & 0 & 0 & \ldots \\
0 & 0 & 0 & 0 & \frac{1}{2} & \frac{1}{2} & \ldots \\
. & . & . & . & . & \ldots
\end{array}\right)
$$

und definieren $B$ als permanente Matrix mit den folgenden Eigenschaften: $b_{l, 2 k}=b_{l, 2 k \nmid 1}$ für alle $k$ und $l$; jede Spalte von $B$ enthält genau ein nichtverschwindendes Element; jede Zeile von $B$ enthält unendlich viele NichtNullen. Nach ERDös-PIRANIAN [4] (Theorem 2) ist kein zeilenfinites reguläres $C$ stärker als $B$. Nach Theorem 4 derselben Arbeit ist kein nicht-zeilenfinites $C$ stärker als $A$. Das vollendet den Beweis, da ja $A$ und $B$ verträglich sind.

\section{Nichtexistenz eines stärkeren Verfahrens bei beschränkten Folgen}

Verschiedene Ergebnisse der allgemeinen Limitierungstheorie zeigen einen Zusammenhang zwischen der Limitierbarkeit beschränkter und unbeschränkter Folgen bei einer Matrix $A$; und dieser Zusammenhang kann zum Nachweis

1) Die Verfasser sind der U. S. National Science Foundation für großzügige Förderung zu Dank verpflichtet (research grants NSF G1014 und NSF G3468 an Wayne State University). 
wesentlicher Eigenschaften von $A$ benützt werden. Zum Beispiel limitiert eine permanente Matrix $A$ gewisse unbeschränkte Folgen, wenn sie eine beschränkt-divergente Folge limitiert. In der vorliegenden Arbeit ergibt der Vergleich von Satz 2 und 3 einen ähnlichen Zusammenhang.

Satz 2. Es gibt zwei permanente, für beschränkte Folgen verträgliche $M a-$ trizen $A$ und $B$, so da $\beta$ in $(m)$ keine stetige Linearform existiert, die in $\mathfrak{A}_{B}$ mit $A-\lim x$ und in $\mathfrak{B}_{B}$ mit $B$-lim $x$ übereinstimmt.

Die Behauptung kann auch anders formuliert werden. Wegen der Verträglichkeit von $A$ und $B$ gibt es eine eindeutige Linearform $L(x)$ in $\mathfrak{A}_{B}+\mathfrak{B}_{B}$ mit $L(x)=A-\lim x$ in $\mathfrak{A}_{B}$ und $L(x)=B \lim x$ in $\mathfrak{B}_{B}$. Aber dieses $L(x)$ ist nicht stetig bezüglich der Normtopologie in $(m)$.

Beweis. Es genügt, zwei permanente Matrizen $A$ und $B$ zu konstruieren, die für beschränkte Folgen verträglich sind und die folgende Singularität aufweisen:

$\left(S_{1}\right)$ Für jedes $\varepsilon>0$ gibt es Folgen $x \in \mathfrak{A}_{B}, y \in \mathfrak{B}_{B}$ mit $\|x-y\|<\varepsilon$ und $|A-\lim x-B-\lim y|>1$.

Der Ausgangspunkt unserer Konstruktion sind die folgenden $(2 \times 3)$-Blocks (mit kleinem $\varrho>0$ ):

$$
A(\varrho)=\left(\begin{array}{llll}
1-\varrho & \varrho & 0 \\
1-\varrho & 0 & \varrho
\end{array}\right), \quad B(\varrho)=\left(\begin{array}{ccc}
\varrho & 0 & 1-\varrho \\
0 & 1 & 0
\end{array}\right) .
$$

Ähnliche Blocks wurden von Petersìn [7] benützt. Die wesentlichen Eigenschaften dieser Blocks sind: Jeder transformiert $(1,1,1)$ in $(1,1)$; weiter führt $A$ das Tripel $(1,0,0)$ in $(1-\varrho, 1-\varrho)$ über und $B$ das Tripel $(1, \varrho, 0)$ in $(\varrho, \varrho)$, so daß zwei benachbarte Tripel in zwei ganz verschiedene (und konstante ${ }^{2}$ )) Paare transformiert werden; wird ein Tripel sowohl von $A$ als auch von $B$ in ein konstantes Paar übergeführt, $\mathrm{mu} ß$ es von der Form $(\sigma, \sigma, \sigma)$ sein. Unter Benützung dieser Blocks ist es leicht, zwei Matrizen $A$ und $B$ zon konstruieren, die die Singularität $\left(S_{1}\right)$ für ein festes $\varepsilon>0$ besitzen.

Für unseren $Z$ weck ist jedoch ein verfeinerter Kondensationsprozeß erforderlich. Mit $A_{n}^{2 m}$ bezeichnen wir eỉnen $(2 m \times 3)$-Block, der jede Zeile von $2^{-n} A\left(4^{-n}\right)$. genau $m$ Male enthält. Die Zeilen werden dabei so angeordnet, $\mathrm{da} B$ in den Zeilen der Matrix

$$
A=\left(\begin{array}{ccccccc}
A_{1}^{2} & 0 & 0 & 0 & 0 & 0 & \ldots \\
0 & A_{1}^{4} & A_{2}^{4} & 0 & 0 & 0 & \ldots \\
0 & 0 & 0 & A_{1}^{8} & A_{2}^{8} & A_{3}^{8} & \ldots \\
. & . & . & . & . & . & \ldots
\end{array}\right)
$$

jede Kombination der Zeilen von $2^{-1} A\left(4^{-1}\right)$ und $2^{-2} A\left(4^{-2}\right)$ zu finden ist, und jede Kombination der Zeilen von $2^{-1} A\left(4^{-1}\right), 2^{-2} A\left(4^{-2}\right), 2^{-3} A\left(4^{-3}\right)$, usw. Ausgehend von $B(\varrho)$ wird die Matrix $B$ in genau derselben Weise definiert.

$A$ und $B$ sind offenbar regulär. Unter Verwendung der Eigenschaften der Blocks erkennen wir, da $B$ es zu jedem $\varrho=4^{-n}$ eine Folge gibt, die aus Tripeln

2) Wir nennen ein Paar $(x, y)$ konstant, wenn $x=y$ gilt. 
$(0,0,0)$ und $(1,0,0)$ besteht sowie $A$-limitierbar zum Werte $2^{-n}(1-g)$ ist, und eine Folge $y$, die in ganz entsprechender Weise sich aus Tripeln $(0,0,0)$ und $(1, \varrho, 0)$ zusammensetzt und von $B$ zum Werte $2^{-n} \varrho$ limitiert wird. Wir haben $\|x-y\|=\varrho=4^{-n}$; und die Differen $z$ von $A-\lim x$ und $B-\lim y$ ist nahe bei $2^{-n}$. Daher besitzen die Matrizen $A, B$ die Singularität $\left(S_{1}\right)$ und wir müssen nur.noch ihre Verträglichkeit nachweisen.

$N_{\dot{p}}$ bezeichne die Menge der natürlichen $Z$ ahlen $k$, für die in der $k$-ten Spalte von $A$ Elemente aus dem Block $A_{p}^{2 m}$ stehen. Die Mengen $N_{p}$ sind paarweise fremd. Nun betrachten wir die "Teiltransformation"

$$
y_{l}^{(p)}=\sum_{k \in N_{p}} a_{l k} x_{k} .
$$

Für jede beschränkte $A$-limitierbare Folge $x$ und jedes $p$ gilt

$$
x_{3 n+1}-x_{3 n+2} \rightarrow 0 \quad \text { für } 3 n \in N_{p} \text { und } n \rightarrow \infty .
$$

Wenn nämlich $\left|x_{3 n+1}-x_{3 n+2}\right|=\delta>0$ für ein festes $n$ mit $3 n \in N_{p}$ gilt, können wir in $A$ zwei Zeilen finden, die beide denselben Block $A_{p}^{2 m}$ treffen und für deren Nummern $l, l^{\prime}$ gilt $\mid y_{l}^{(p)}-y_{l^{(p)}}^{(p)}=2^{-p} 4^{-p} \delta$. Wegen der bei der Konstruktion von $A$ benützten Vorschrift über die Kombination der Zeilen aus den Blocks haben wir dann in $A$ auch zwei Zeilen, die wiederum denselben Block $A_{p}^{2 m}$ treffen und für deren Nummern $\lambda, \lambda^{\prime}$ gilt $\left|y_{\lambda}-y_{\lambda^{\prime}}\right|=\left|y_{l}^{(p)}-y_{l^{\prime}}^{(p)}\right|$. Daher würde aus $\overline{\lim }\left|x_{3 n+1}-x_{3 n+2}\right|=\delta>0$ (für $3 n \in N_{p}$ und $n \rightarrow \infty$ ) folgen, da $\beta$ die Oszillation der $A$-Transformierten von $x$ mindestens $8^{-p} \delta$ ist, im Widerspruch zur $A$-Limitierbarkeit von $x$.

Wenn nun die Folge $x$ auch $B$-limitierbar ist, so erhalten wir in ähnlicher Weise, unter Benutzung von (4) und der Eigenschaften von $B(\varrho)$,

$$
x_{3 n}-x_{3 n+2} \rightarrow 0 \text { für } 3 n \in N_{p} \text { und } n \rightarrow \infty \text {. }
$$

Daher gilt für jedes $p$

Überdies ist

$$
\sum_{k \in N_{p}}\left(a_{l k}-b_{l k}\right) x_{k} \rightarrow 0 \quad \text { für } l \rightarrow \infty .
$$

$$
\sum_{k \notin N_{1}, \ldots, N_{r}}\left(\left|a_{l k}\right|+\left|b_{l k}\right|\right) \leqq 2^{-r+1} \quad(l=0,1, \ldots),
$$

so daß wegen (5) und (6) für jedes $r$

$$
\begin{aligned}
\left|\sum_{k=0}^{\infty}\left(a_{l_{k}}-b_{l k}\right) x_{k}\right| & \leq\left|\sum_{k \in N_{1}, \ldots, N_{r}}\left(a_{l k}-b_{l k}\right) x_{k}\right|+2^{-r+1}\|x\| \\
& =o(1)+2^{-r+1}\|x\| \quad(l \rightarrow \infty)
\end{aligned}
$$

gilt, was die Verträglichkeit von $A$ und $B$ für beschränkte Folgen beweist.

\section{Die Formulierung der Verträglichkeitssätze}

Die in Satz 2 konstruierte Singularität kann nur vorkommen, wenn die Verfahren $A$ und $B$ unverträglich sind:

Satz 3. Zwei permanente Matrixverfahren $A$ und $B$ sind unverträglich, wenn sie die Singularität $\left(S_{1}\right)$ besitzen. 
Wenn $A$ und $B$ verträglich sind (für alle Folgen), können wir dje Existenz einer stetigen Linearform $L(x)$ in $(m)$ zeigen, die $A-\lim x$ und $B-\lim x$ auf $\mathfrak{A}_{B}+\mathfrak{B}_{B}$ fortsetzt. Darüber hinaus gilt sogar:

Satz 4. Sind $A$ und $B$ permanente verträgliche Matrixverfahren, so gibt es ein permanentes Matrixverfahren $C$, das mit $A$ und $B$ für beschränkte Folgen verträglich ist und dessen Wirkfeld $\mathfrak{A}_{B}+\mathfrak{B}_{B}$ enthält.

Satz 3 wird sich aus dem untenstehenden Lemma 2 ergeben, während der Beweis von Satz 4 zusätzlich die Betrachtung gewisser konvexer Mengen erfordert.

\section{Einige Lemmata}

Wir beginnen mit einem einfachen Lemma, das besagt, da $\beta$ permanente Matrizen zu zeilen- und spaltenfiniten Matrizen ,gestutzt" werden können, ohne ihr Verhalten bezüglich beschränkten Folgen zu ändern.

Lemma 1. $A$ und $B$ seien permanente Matrizen. Dann gibt es eine Folge $\varrho_{k} \nearrow \infty$ und zwei Folgen natürlicher Zahlen $p_{l} \nearrow \infty, r_{l} \nearrow \infty$ mit $p_{l}<r_{l}$, so da $\beta$ folgendes gilt. Sind $A^{\prime}, B^{\prime}$ die Matrizen, die aus $A, B$ entstehen, indem man die Elemente $a_{l}, b_{l_{k}}$ mit $k<p_{l}$ und die mit $k \geq r_{l}$ durch Null ersetzt, so bestehen für jede Folge $x_{k}$ mit $x_{k}=O\left(\varrho_{k}\right)$ die Beziehungen

$$
x_{l}^{A}-x_{l}^{A^{\prime}} \rightarrow 0, \quad x_{l}^{B}-x_{l}^{B^{\prime}} \rightarrow 0 \quad(l \rightarrow \infty) .
$$

Beweis. Es genügt, die Zahlen $\varrho_{k}$ so langsam gegen $\infty$ gehen zu lassen, daß alle Reihen $\sum_{k}\left|a_{l_{k}}\right| \varrho_{k}, \sum_{k}\left|b_{l k}\right| \varrho_{k}$ konvergieren, und dann $p_{l}, r_{l}$ so zu
wählen, daß

$$
\sum_{k<p_{l}}\left|a_{l k}\right| \varrho_{k} \rightarrow 0, \quad \sum_{k \geq n_{l}}\left|a_{l k}\right| \varrho_{k} \rightarrow 0 \quad(l \rightarrow \infty)
$$

gilt, sowie die entsprechenden Beziehungen mit $b_{l k}$ statt $a_{l k}$ bestehen.

Lemma 2. $A$ und $B$ seien permanente Matrixverfahren und $\varrho_{k}, p_{l}, r_{l}$ geniä $\beta$ Lemma 1 geweählt. Weiter mögen $A$ und $B$ die folgende Singularität besitzen: $\left(S_{2}\right) Z u$ jedem $\varepsilon>0$. gibt es ein $M=M(\varepsilon)$ und ein $m=m(\varepsilon)$ derart, daß fïr jedes $n>m$ zwei Folgen $x=x(\varepsilon, n), y=y(\varepsilon, n)$ existieren mit den Eigenschaften

$$
\begin{gathered}
\|x\|+\|y\|<M, \\
\left|x_{k}+y_{k}-1\right|<\varepsilon \quad \text { für } m \leq k<n, \\
\left|x_{l}^{A}\right|+\left|y_{l}^{B}\right|<\varepsilon \quad \text { fiur alle } l \text { mit } m \leq p_{l}, r_{l}<n .
\end{gathered}
$$

Dann sind $A$ und $B$ nicht verträglich, nicht einmal für Folgen $x_{k}=O\left(\varrho_{k}^{*}\right)$ bei beliebig vorgegebenen $\varrho_{k}^{*} \nearrow \infty$.

Bemerkung. Erfüllen zwei Folgen $x, y$ mit $a=A$-lim $x$ und $b=B$-lim $y$ die Eigenschaft $\left(S_{1}\right)$ für ein bestimmtes $\varepsilon>0$, so befriedigen die Folgen $x^{\prime}=(x-a) /(b-a), y^{\prime}=(b-y) /(b-a)$ die in $\left(S_{2}\right)$ genannten Ungleichungen für dasselbe $\varepsilon$ und beliebiges $n$, wenn wir nur $M$ und $m$ geeignet wählen $\left[M\right.$ so groß, daß (8), $m$ so groß, daß (10) gilt]. Daher ist $\left(S_{1}\right)$ eine stärkere Singularität als $\left(S_{2}\right)$ und somit folgt Satz 3 aus Lemma 2. 
Beweis. Wir dürfen $\varrho_{k}^{*} \leq \varrho_{k}$ voraussetzen, und wegen Lemma 1 können wir annehmen, daß $a_{l k}=b_{l k}=0$ für $k<p_{l}$ und für $k \geqq \gamma_{l}$ ist. Wir wählen eine Folge von natürlichen Zahlen $0<q_{1}<q_{2}<\cdots<q_{i}<\cdots$, die so rasch wächst, daß jedes Intervall $\left\langle p_{l}, r_{l}\right\rangle$ höchstens zwei der Intervalle $\left\langle q_{i}, q_{i+1}\right\rangle$ trifft.

Wir wählen Zahlen $\varepsilon_{j} \succ 0$ und erhalten gemäß Lemma 2 Zahlen $M_{j}=M\left(\varepsilon_{j}\right)$, von denen wir monotones Wachstum voraussetzen dürfen. Dann bestimmen wir Zahlen $m_{j}$, die eine monotone Teilfolge der $q_{i}$ bilden, auf induktiven Wegen. Wir wählen $m_{j}=q_{i(j)} \geq m\left(\varepsilon_{j}\right)$ so groß, daß

$$
\begin{gathered}
M_{j} \leq Q_{k}^{*} \quad \text { für } k \geq m_{j}, \\
\frac{M_{j}}{s(j)-1}<\varepsilon_{j} \quad[\text { mit } s(j)=i(j+1)-i(j)>1]
\end{gathered}
$$

gilt. Dann setzen wir $n_{j}=m_{j+2}$. Zu diesen $\varepsilon_{j}, M_{j}, m_{j}, n_{j}$ gibt es gemäß $\left(S_{2}\right)$ [Formeln (8) bis (10)] passende Folgen $x^{(j)}, y^{(j)}$. Wir möchten aus diesen zwei (möglicherweise unbeschränkte) Folgen $x, y$ zusammenstellen, die die Unverträglichkeit von $A$ und $B$ darlegen. Eine direkte Interpolation $x_{k}=x_{k}^{(i)}$ für $m_{j} \leqq k<m_{j+1}$ würde nicht zum Ziele führen, da wir nun nicht zeigen könnten, daß

$$
x_{l}^{A}=\sum_{p_{l} \leq k<n_{l}} a_{l k} x_{k}
$$

auch dann klein ist, wenn ein $m_{j}$ im Intervall $\left\langle p_{l}, r_{l}\right\rangle$ liegt.

Diese Schwierigkeit beseitigen wir durch einen Glättungsprozeß. Sei

$$
\mathfrak{z}^{\prime}=\frac{i-i(j)}{s(j)-1} \quad \text { für } k \in\left\langle q_{i}, q_{i+1}\right)<\left\langle m_{j}, m_{j+1}\right),
$$

also $0 \leqq w_{k} \leqq 1$. Wir setzen

$$
x_{k}=\left\{\begin{array}{l}
\frac{1}{2} \quad \text { für } 0 \leqq k<m_{1} \\
\left(1-w_{k}\right) x_{k}^{(j-1)}+w_{k} x_{k}^{(j)} \quad \text { für } m_{j} \leq k<n_{j+1}
\end{array}\right.
$$

und definieren die $y_{k}$ entsprechend, mit $y_{k}^{(i)}$ an Stelle von $x_{k}^{(i)}$. Wegen (11) und (14) ist dann $\left|x_{k}\right| \leqq \varrho_{k}^{*},\left|y_{k}\right| \leqq \varrho_{k}^{*}$ für $k \geq m_{1}$.

Zum Nachweis der Kleinheit von $x_{l}^{A}$ wählen wir $i$ und $j$ so, daß

$$
\left\langle p_{l}, r_{l}\right\rangle<\left\langle q_{i-1}, q_{i+1}\right\rangle \text { und }\left\langle q_{i}, q_{i+1}\right)<\left\langle m_{j}, m_{j+1}\right)
$$

st. Im Falle $q_{i}=m_{j}$ (und $j>1$ ) gilt $x_{k}=x_{k}^{(j-1)}\left(\right.$ für $k$ in $\left\langle q_{i-1}, q_{i+1}\right)$ ) und daher $\left|x_{i}^{A}\right|=\left|x_{l}^{j-1}(A)\right|<\varepsilon_{j-1}$ wegen $m_{j-1}<q_{i-1} \leq p_{l}<r_{l}<q_{i+1}<m_{j+1}=n_{j-1}$. Ist aber $q_{i}>m_{1}$, so gilt $x_{k}=\left(1-w_{k}\right) x_{k}^{(i-1)}+w_{k} x_{k}^{(j)}$ (für $k$ in $\left\langle q_{i-1}, q_{i+1}\right)$ ), wobei sich $w_{k}$ von $w=w_{q_{i}}$ um höchstens $1 /(s(j)-1)<\varepsilon_{j} / M_{j}$, unterscheidet. Also unterscheidet sich $x_{l}(A)$ von

$$
\sum_{p_{l} \leq k<r_{l}} a_{l k}\left\{(1-w) x_{k}^{(j-1)}+w x_{k}^{(j)}\right\}=(1-w) x_{l}^{(i-1)}(A)+w x_{l}^{(j)}(A)
$$

um höchstens $2 \varepsilon_{j} \sum_{k}\left|a_{l k}\right| \leqq \Omega \varepsilon_{j}$. Wegen $m_{j-1} \leqq p_{l}<r_{l}<n_{j-1}$ haben wir $\left|x_{i}^{(j-1)}(A)\right|<\varepsilon_{j-1}$. Ebenso folgt $\left|x_{l}^{(j)}(A)\right|<\varepsilon_{j} \leq \varepsilon_{j-1}$. Daher gilt in jedem 
Fall $\left|x_{i}^{A}\right| \leq \varepsilon_{j-1}+\Omega \varepsilon_{j} \rightarrow 0$ für $l \rightarrow \infty$. Genauso zeigen wir $y_{l}^{B} \rightarrow 0$. Da ja $x_{k}+y_{k} \rightarrow 1$ gilt, haben wir $x_{l}^{B} \rightarrow 1$, so daß $A$ und $B$ die Folge $x$ zu verschiedenen Werten limitieren. Das vollendet den Beweis.

Enthält das Wirkfeld einer (permanenten) Matrix $A$ für beliebig gegebenes $\varrho_{k} \nearrow \infty$ Folgen $x_{k}=O\left(\varrho_{k}\right)$, so enthält es auch gewisse beschränkt-divergente Folgen (WILANSKY-ZeLLer [8]). Im Gegensatz dazu können zwei (permanente) Matrizen $A$ und $B$, wie Satz 2 und Lemma 2 zeigen, für beschränkte Folgen verträglich, für beliebig langsam wachsende Folgen aber unverträglich sein.

Bemerkung. Lemma 2 kann auf eine beliebige endliche Anzahl von Verfahren erweitert werden. Für drei Verfahren $A, B, C$ zum Beispiel bedeutet die Singularität $\left(S_{2}\right)$ die Existenz dreier Folgen $x(\varepsilon, n), y(\varepsilon, n), z(\varepsilon, n)$, die folgende Bedingungen erfüllen: $\|x\|+\|y\|+\|z\|<M ;\left|x_{k}+y_{k}+z_{k}-1\right|<\varepsilon$ für $m \leqq k<n ;\left|x_{l}^{A}\right|+\left|y_{l}^{B}\right|+\left|z_{l}^{C}\right|<\varepsilon$ für $m \leqq p_{l}, r_{l}<n$. Die Folgerung von Lemma 2 ist dann die Existenz von drei Folgen $x, y, z$ beliebig langsamen Wachstums, die beziehungsweise $A-, B$-, $C$-limitierbar zum Werte Null sind, aber $x_{k}+y_{k}+z_{k} \rightarrow 1$ erfüllen.

\section{Beweis des Satzes 4}

Wir dürfen annehmen, daß $A, B$ die Eigenschaften der Matrizen $A^{\prime}, B^{\prime}$ aus Lemma 1 besitzen (also ,gestutzt" sind). Da $A$ und $B$ verträglich sind, ist die Voraussetzung von Lemma 2 nicht erfüllt. Daher gibt es ein $\varepsilon>0$, so daß zu jedem Paar $M, m$ ein $n$ existiert mit der Eigenschaft, daß (8), (9), (10) nicht gleichzeitig erfüllt sein können. Wir nehmen $M=m$ und bezeichnen mit $n(m)$ das entsprechende $n$.

Wir bemerken zunächst: Ist $X$ ein Vektorraum und $\varrho(x, y)$ eine Halbnorm in $X \times X$, so ist $\varrho(z)=\inf _{z=x+y} \varrho(x, y)$ eine Halbnorm in $X$. - Im endlichdimensionalen Raum $R(m)$ der Folgen $z=\left\{0, \ldots, 0, z_{m}, z_{m+1}, \ldots, z_{n-1}, 0, \ldots\right\}$ [wo $n=n(m)]$ definieren wir

$$
\varrho_{m}(z)=\inf _{x+y=z}\left[\frac{1}{\varepsilon} \sup _{m \leqq p l, n<n}\left\{\left|x_{l}^{A}\right|+\left|y_{l}^{B}\right|\right\}+\frac{1}{M} \sup _{m \leq k<n}\left\{\left|x_{k}\right|+\left|y_{k}\right|\right\}\right] .
$$

Dieser.Ausdruck ist eine Norm in $R(m)$. Wegen der Wahl von $n$ gehört die Folge $z_{0}=\left\{z_{0 k}\right\}$ mit $z_{0 k}=1$ für $m \leq k<n$ und $=0$ sonst nicht zu der Einheitskugel $\varrho_{m}(z)<1$ in $R(m)$. Nach dem Hahn-Banachschen Theorem existiert daher in $R(m)$ (versehen mit der Norm $\varrho_{m}$ ) eine Linearform

mit $\left\|L_{m}\right\| \leqq 1$ und $L\left(z_{0}\right)=1$.

$$
L_{m}(z)=\sum_{m \leq k<n} c_{m k} z_{k}
$$

Wenn wir nun zusätzlich $c_{m k}=0$ für $k<m$ und für $k \geqq n$ setzen, so hat die Matrix $C=\left\{c_{l k}\right\}$ die Eigenschaften $\sum_{k} c_{l k}=1$ für $l=0,1, \ldots$ und $\lim _{l} c_{l k}=0$ für $k=0,1, \ldots$ Sei $z$ eine beschränkte Folge, die von $A$ zu Null limitiert wird. Dann ist $\left|z_{l}^{A}\right|<\delta$ für alle großen $l$; und für die Projektion $\bar{z}$ von $z$ auf 
$R(m)$ gilt $\varrho_{m}(\bar{z}) \leq \frac{\delta}{\varepsilon}+\frac{\|z\|}{m}$ für alle großen $m$ wegen (16). Somit haben wir

$$
\left|\sum_{k} c_{m k} z_{k}\right|=\left|L_{m}(\bar{z})\right| \leq \frac{\delta}{\varepsilon}+\frac{\|z\|}{m},
$$

so $\mathrm{daB} z$ von $C$ zum Werte Null limitiert wird.

Weiter folgt nun, daß $C$ permanent ist und jede beschränkte $A$-limitierbare (oder auch $B$-limitierbare) Folge limitiert. SchlieBlich ist $C$ nach einem allgemeinen Satz von Brudno [2] (s. auch Petersen [6]) mit $A$ und $B$ verträglich für beschränkte Folgen.

Satz 4 kann auf eine beliebige endiche Anzahl von Verfahren ausgedehnt werden, wenn die Verträglichkeit geeignet definiert wird. Zum Beispiel nennen wir drei Verfahren $A, B, C$ simultan verträglich, wenn aus $x+y+z=0$ mit $x \in \mathfrak{A}, y \in \mathfrak{B}, z \in \mathbb{C}$ stets folgt $A-\lim x+B-\lim y+C-\lim z=0$; d.h. wenn es eine (nicht notwendig stetige) Linearform in $\mathfrak{A}+\mathfrak{B}+\mathbb{C}$ gibt, die die $A-, B$ und $C$-Limites fortsetzt. Wir formulieren:

Satz $4^{*}$. Sind die permanenten Matrixverfahren $A, B, C$ simultan verträglich, so gibt es ein permanentes Matrixverfahren $D$, das für beschränkte Folgen stärker als und verträglich mit $A, B, C$ ist.

Der Beweis bleibt im wesentlichen derselbe wie bei Satz 4, unter geeigneter Abänderung der Definition der Normen (16) und Verwendung der in Abschnitt 5 erwähnten Variante von Lemma 2.

Es ist zu erwähnen, daß $A, B, C$ paarweise verträglich sein können, ohne simultan verträglich zu sein. Drei von PeTERsen [7] konstruierte Verfahren, die $\mathfrak{A}_{B}+\mathfrak{B}_{B}+\mathfrak{C}_{B}=(m)$ erfüllen, weisen diese Singularität auf.

Natürlich ist für Satz 4 die Voraussetzung der Verträglichkeit wesentlich. Dies zeigen einmal allgemeine Verträglichkeitssätze (BRUdno [2], PETERSEN [6]), zum andern die beiden durch $A-\lim x=\lim x_{2 k}, B-\lim x=\lim x_{2 k+1}$ definierten Verfahren, bei denen $\mathfrak{A}+\mathfrak{B}$ alle Folgen umfaßt und $\mathfrak{A}_{B}+\mathfrak{B}_{B}=(m)$ ist.

\section{Starke Limitierbarkeit}

Die Methode aus Abschnitt 5 und 6 läßt sich auch bei starker Limitierbarkeit anwenden.

Satz 5. Zwei permanente positive Matrizen $A, B$ sind genau dann stark verträglich, wenn es eine permanente positive Matrix $C$ gibt, die jede stark $A$ limitierbare und jede stark B-limitierbare Folge zum selben Wert stark limitiert.

Lemma 3. Wenn es zal zwei permanenten positiven Matrizen $A$ und $B$ bei jedem $\varepsilon>0$ eine natürliche $Z$ ahl $m=m(\varepsilon)$.gibt, so daß bei beliebig großem $n$ zwei positive Folgen $x, y$ existieren mit

$$
\begin{array}{ll}
x_{k}+y_{k}=1 & (m \leq k<n) \\
x_{l}^{A}+y_{l}^{B}<\varepsilon & (l=0,1, \ldots),
\end{array}
$$

so sind $A$ und $B$ nicht stark verträglich.

Bew่eis. Wir wählen eine Folge $\varepsilon_{j}>0$ mit $\sum \varepsilon_{j}<\infty$. Sei $m_{j}=m\left(\varepsilon_{j}\right)$; wir dürfen dabei $m_{j}<m_{j+1}$ annehmen. Dann setzen wir $n_{i}=m_{j+1}$. Es gibt 
positive Folgen $x^{(j)}$ und $y^{(j)}$, die (17) und (18) mit diesen $\varepsilon_{j}, m_{j}, n_{j}$ erfüllen. Wir dürfen $x_{k}^{(j)}=0$ für $k$ außerhalb $\left\langle m_{j}, n_{j}\right\rangle$ annehmen. Wir setzen

$$
x_{k}= \begin{cases}0 & \text { für } k<m_{1}, \\ x_{k}^{(j)} & \text { für } m_{j} \leq k<n_{j} \quad(j=1,2, \ldots),\end{cases}
$$

und definieren $y_{k}$ entsprechend. Dann gilt für jedes feste $p$

$$
x_{l}^{A}=\sum_{j=1}^{p-1} x_{i}^{(j)}(A)+\sum_{j=p}^{\infty} x_{i}^{(j)}(A) \leq o(1)+\sum_{j=p}^{\infty} \varepsilon_{j} \quad(l \rightarrow \infty),
$$

also $x_{l}^{A} \rightarrow 0$. Ebenso erhalten wir $y_{l}^{B} \rightarrow 0$.. Daher ist $x$ stark $A$-limitierbar zum Werte 0 (alle vorkommenden Glieder sind ja nichtnegativ), und somit $\left\{1-x_{k}\right\}$ stark $B$-limitierbar zum Werte Null, also $x$ stark $B$-limitierbar zum Werte 1 und daher $A$ und $B$ nicht stark verträglich.

Beweis von Satz 5. Wir müssen nur die Notwendigkeit der Bedingung beweisen. $A$ und $B$ seien stark verträglich. Dann ist die Voraussetzung von Lemma 3 nicht erfüllt. Daher gibt es ein $\varepsilon>0$, so daß für jedes $m$ ein $n=n(m)$ existiert mit der Eigenschaft, da $\beta$ für positive Folgen $x, y$ die Bedingungen (17) und (18) nicht gleichzeitig erfüllt sein können. Im Raume $R(m)$ der reellen Folgen $z=\left\{0, \ldots, 0, z_{m}, z_{m+1}, \ldots, z_{n-1}, 0, \ldots\right\}$, den wir in natürlicher Weise als Vektorverband auffassen, definieren wir die Halbnorm

$$
\left.\varrho_{m}(z)=\inf _{\substack{|z|=x+y \\ x, y \geq 0}}\left[\frac{1}{\varepsilon} \sup _{l=0,1, \ldots}\left(x_{l}^{A}+y_{l}^{B}\right)\right] \quad \text { [wo } x, y \in R(m)\right] .
$$

Sei $z_{0}=\left\{z_{0 k}\right\}$ mit $z_{0 k}=1$ für $m \leq k<n$ und $=0$ sonst. Ist $z \geq z_{0}$ in $R(m)$, dann gehört $z$ nicht der durch $\varrho_{m}(z)<1$ gegebenen Einheitskugel $U$ an. Denn im Falle $z \in U$ gäbe es $x, y \geq 0$ in $R(m)$ mit $x+y=z$ und $x_{l}^{A}+y_{l}^{B}<\varepsilon$ $(l=0,1, \ldots)$; und wir könnten $x^{\prime}, y^{\prime} \in R(m)$ mit $0 \leq x^{\prime} \leq x, 0 \leq y^{\prime} \leq y$ und $x^{\prime}+y^{\prime}=z_{0}$ finden. Dann wäre $x_{l}^{\prime}(A)+y_{l}^{\prime}(B)<\varepsilon$ für alle $l$ und somit $z_{0} \in U$, im Widerspruch zur Definition von $\varepsilon$.

Sei $K$ der Kegel (in $R_{m}$ ) aller Elemente $z \geq z_{0}$. Wir können $U$ und $K$ in $R_{m}$ durch eine Hyperebene $H$ trennen, die eine Stützhyperebene für $K$ ist (Bourbaki [1, S. 71]). Dann muß $H$ den Punkt $z_{0}$ enthalten. Úberdies enthält $H$ nicht den Ursprung, da sonst der im Innern von $K$ gelegene Punkt $\bar{z}$ (wo $\bar{z}_{k}=2$ für $m \leq k<n$ ) zu $H$ gehören würde. Daher können wir die Gleichung von $H$ in der Form $L_{m}(z)=1$ schreiben, wo

$$
L_{m}(z)=\sum_{m \leq k<n} c_{m k} z_{k} \text {. }
$$

Da $L_{m}(z) \geq 1$ in $K$ und $L_{m}\left(z_{0}\right)=1$, ist hierbei $c_{m k} \geq 0$ und $\sum_{k} c_{m k}=1$. Wie in Abschnitt 5 ergänzen wir nun die Definition von $C$ und vollenden den Beweis.

\section{Kern-Verträglichkeit}

Wir schließen mit einem Ergebnis, das Satz 4 auf Kerne überträgt. Der zugrunde gelegte Raum, in dem die Trennung konvexer Mengen durchgeführt wird, ist nun der Raum $\left(l^{1}\right)$ der komplexen Folgen $x=\left\{x_{k}\right\}$ mit $\|x\| \doteq \sum_{k}\left|x_{k}\right|<+\infty$,
der jedoch mit reellen Skalaren versehen wird. 
Satz 6. Für zwei permanente Matrixverfahren $A$ und $B$ kann genau eine der folgenden Möglichkeiten auftreten:

a) $A$ und $B$ sind nicht kern-verträglich für beschränkte Folgen.

b) Es gibt eine permanente Matrix $C$, die für beschränkte Folgen kern-stärker als $A$ und $B$ ist.

Beweis. Die $l$-ten Zeilen $a^{(l)}, b^{(l)}$ von $A$ und $B$ betrachten wir als Elemente in $\left(l^{1}\right)$. Mit $R_{n}, R_{n}^{\prime}$ bezeichnen wir die konvexen Hüllen der Mengen $a^{(l)}(l \geq n)$ bzw. $b^{(l)}(l \geq n)$. Es gibt zwei Fälle.

a) Der Abstand $\varrho\left(R_{n}, R_{n}^{\prime}\right)$ ist ungleich Null für ein bestimmtes $n$. Dann gibt es ein $\delta>0$, so daß $\varrho\left(R_{n}, R_{n}^{\prime}\right) \geq 2 \delta$ ist für alle großen $n$. Wir werden zeigen. $\mathrm{daB}$ für eine geeignete stetige Linearform $L(x)$ in $\left(l^{1}\right)$ die folgenden Beziehungen gelten: $L(x) \leq \lambda$ in $R_{n}$ und $L(x) \geq \lambda+\varepsilon$ in $R_{n}^{\prime}$ (mit festem $\varepsilon>0$ ). Sei $R_{n}^{\prime \prime}$ die $\delta$-Umgebung von $R_{n}^{\prime}$. Da $R_{n}^{\prime \prime}$ eine offene konvexe Menge ist, gibt es nach einem Trennungssatz (Bourbaki [1, S. 71]) eine abgeschlossene Hyperebene $H$ mit Gleichung $L(x)=\lambda$, die $R_{n}$ und $R_{n}^{\prime \prime}$ trennt: $L(x) \leq \lambda$ in $R_{n}, L(x) \geq \lambda$ in $R_{n}^{\prime \prime}$. Die Hyperebene $H$ hat einen Abstand $\geq \delta$ von $R_{n}^{\prime}$; somit gibt es eine parallele Hyperebene $L(x)=\lambda+\varepsilon$ (mit geeignetem $\varepsilon>0$ ), die $H$ und $R_{n}^{\prime}$ trennt. Wir haben $L(x) \geq \lambda+\varepsilon$ in $R_{n}^{\prime}$.

Die Linearform $L(x)$ gestattet eine Darstellung $L(x)=\sum_{k}\left(\sigma_{k} \xi_{k}+\tau_{k} \eta_{k}\right)$, wo $x_{k}=\xi_{k}+i \eta_{k}$ und $\sigma_{k}, \tau_{k}$ beschränkte Folgen sind. Mit $s=\sigma-i \tau$ erfüllen dann die Realteile von $s_{l}^{A}, s_{l}^{B}$ die Ungleichungen $\operatorname{Re} s_{l}^{A} \leq \lambda$ und $\operatorname{Re} s_{l}^{B} \geq \lambda+\varepsilon$ für $l \geq n$. Daher sind $A$ - und $B$-Kern von $s$ fremd.

b) Sei $\varrho\left(R_{n}, R_{n}^{\prime}\right)=0$ für jedes $n$. Dann können wir für jedes $n$ Elemente $c^{(n)}=\left\{c_{n k}\right\}$ und $d^{(n)}=\left\{d_{n k}\right\}$ auswählen, so $\mathrm{daB} c^{(n)} \in R_{n},\left\|d^{(n)}\right\|<n^{-1}$. und $c^{(n)}+d^{(n)} \in R_{n}^{\prime}$ gilt. Wir bilden die Matrix $C=\left\{c_{n k}\right\}$. Da $c^{(n)}$ in der konvexen Hülle von $a^{(n)}, a^{(n+1)}, \ldots$ liegt, sehen wir leicht, daB $C$ eine permanente Matrix und für beschränkte Folgen kern-stärker als $A$ ist. Im selben Sinne ist $C+D$ stärker als $B$. Da aber $D$ alle beschränkten Folgen zu Null limitiert, gilt diese Stärkebeziehung auch zwischen $C$ und $B$, w. z. b. w.

\section{Literatur}

[1] Bourbaki, N.: Elements de Mathematique, Livre V, Chap. I, II. Paris 1953. [2] Brudno, A. L.: Summation of bounded sequences. Mat. Sbornik, N. S. 16, 191-247 (1945). - [3] Brudno, A. L.: Die Normen von Toeplitzschen Feldern. Doklady Akad. Nauk SSSR., N. S. 81, 11 -14 (1953). - [4] Erdös, P., and G. Piranian: Convergence fields of row-finite and row-infinite Toeplitz transformations. Proc. Amer. Math. Soc. 1, 397-401 (1950). - [5] Lorentz, G. G.; and A. Robinson: Core-consistency and total inclusion for methods of summability. Canadian J. of Math. 6, 27-34 (1953). [6] Petersen, G. M.: Summability methods and bounded sequences. J. London Math. Soc. 31, 324-326 (1956). - [7] Petersen, G. M.: Consistent summability methods. J. London Math. Soc. 32, 62-65 (1957). - [8] Wilansky, A., u. K. Zeller: The inverse matrix in summability: reversible matrices. J. London Math. Soc. (im Druck).

University of Michigan (Ann Arbor, Mich.) und Wayne State University (Detroit 2,.Mich.)

(Eingegangen am 20. Juni 1957) 